\title{
Contrasting patterns of clonality and fine-scale genetic structure in two rare sedges with differing geographic distributions
}

\author{
RM Binks, MA Millar and M Byrne
}

For plants with mixed reproductive capabilities, asexual reproduction is more frequent in rare species and is considered a strategy for persistence when sexual recruitment is limited. We investigate whether asexual reproduction contributes to the persistence of two co-occurring, rare sedges that both experience irregular seed set and if their differing geographic distributions have a role in the relative contribution of clonality. Genotypic richness was high $(R=0.889 \pm 0.02)$ across the clustered populations of Lepidosperma sp. Mt Caudan and, where detected, clonal patches were small, both in ramet numbers $(\leqslant 3$ ramets/genet) and physical size $(1.3 \pm 0.1 \mathrm{~m})$. In contrast, genotypic richness was lower in the isolated $L$. sp. Parker Range populations, albeit more variable $(R=0.437 \pm 0.13)$, with genets as large as 17 ramets and up to $5.8 \mathrm{~m}$ in size. Aggregated clonal growth generated significant fine-scale genetic structure in both species but to a greater spatial extent and with additional genet-level structure in L. sp. Parker Range that is likely due to restricted seed dispersal. Despite both species being rare, asexual reproduction clearly has a more important role in the persistence of $L$. sp. Parker Range than $L$. sp. Mt Caudan. This is consistent with our prediction that limitations to sexual reproduction, via geographic isolation to effective gene exchange, can lead to greater contributions of asexual reproduction. These results demonstrate the role of population isolation in affecting the balance of alternate reproductive modes and the contextual nature of asexual reproduction in rare species.

Heredity (2015) 115, 235-242; doi:10.1038/hdy.2015.32; published online 15 April 2015

\section{INTRODUCTION}

Many plants utilize a combination of sexual and asexual reproduction and the balance between these strategies varies widely within and among taxa (Eckert, 2002). Although sexual reproduction produces new genetic combinations with each event and facilitates the connectivity of populations through specialized dispersal mechanisms, asexual reproduction generally produces genetically identical offspring that are unlikely to disperse far from the parent plant (Eckert, 2002). Because of these contrasts, the relative contributions of sexual and asexual reproduction can have great impacts on the genetic diversity and structure of populations (reviewed in Ellstrand and Roose, 1987; Eckert, 2002; Balloux et al., 2003; Vallejo-Marín et al., 2010).

Because of the limited dispersal capacity of asexual reproduction and because species with dual reproduction tend to form multiclonal populations, the greatest genetic impact of clonality occurs at fine spatial scales within populations (Vallejo-Marín et al., 2010). Specifically, this impact is influenced by the size and spatial architecture of genets within populations. Size can relate to the number of ramets per genet and frequency distributions within clonal populations are typically leptokurtic: populations tend to be represented by a few large genets with many smaller genets, resulting in disproportionate genetic contributions to the reproductive effort of the population (Vallejo-Marín et al., 2010). Moreover, the greater the number of ramets, the smaller the effective population size of a given population relative to its apparent census population size. Consequently, genetic diversity and population viability can be significantly overestimated in census counts without knowledge of clonal extent (Rossetto et al., 2004). In addition, the spatial size and arrangement of genets varies according to the pattern of clonal growth and affects fine-scale spatial genetic structure (Vallejo-Marín et al., 2010). Short rhizomes result in aggregated clusters of ramets within genets (that is, phalanx growth strategy) while longer rhizomes can spread in multiple directions over longer distances (that is, guerrilla growth strategy) such that different genets may intersect (Lovett Doust, 1981). Aggregated phalanx growth not only results in stronger patterns of fine-scale genetic structure but also increases the chances of geitonogamous selfing, particularly with increasing genet size, and consequently increases the risk of inbreeding depression (Charpentier, 2002; Albert et al., 2008; Ohsako, 2010).

Asexual reproduction is considered favourable when sexual reproduction is absent or irregular. Through asexual propagation, individuals effectively increase in size and boost the number of inflorescences produced, allowing rapid expansion and on-going persistence, while maximizing the chances of successful sexual events that may otherwise be unlikely (Honnay and Bossuyt, 2005; VallejoMarín et al., 2010). It follows then that the balance between sexual and asexual reproduction often becomes skewed toward increased asexual reproduction in populations or species that are rare or occur in marginal habitats, where factors such as resource limitation, 
environmental extremes, geographic isolation or genetic incompatibilities may limit successful sexual reproduction (Eckert, 2002; Honnay and Bossuyt, 2005; Silvertown, 2008). Despite the benefits of asexual reproduction, extensive clonality can also have broad fitness costs, such as inbreeding depression, the accumulation of deleterious mutations and reduced genotypic diversity (Balloux et al., 2003). Thus, while asexuality may be favourable to rare species for persistence in the short term, if the factors limiting sexual reproduction are prolonged or exacerbated, clonal populations may be poorly equipped to adapt to environmental change (Balloux et al., 2003; Honnay and Bossuyt, 2005; Silvertown, 2008; Meloni et al., 2013). Moreover, given the prevalence of clonal to sexual reproduction in rare species (Silvertown, 2008), which are already vulnerable to extinction due to loss of diversity and inbreeding depression (Ellstrand and Elam, 1993), knowledge of the frequency, spatial dynamics and fine-scale genetic impacts of asexual propagation is crucial to applying effective conservation management.

The south-west of Western Australia is a global biodiversity hotspot with a remarkable diversity of flora (Myers et al., 2000). Geological stability across this ancient landscape, coupled with Pleistocene climatic fluctuations and complex soil mosaics (Hopper, 1979), have led to an array of common and rare species that show complex evolutionary patterns of fragmentation and structuring (Byrne et al., 2014). Lepidosperma is one of the five largest genera in this region, consisting of many rare species with narrow geographic ranges that are associated with specific geologies (Barrett, 2013). Lepidosperma are known to exhibit rhizomatous propagation (Barrett, 2013), which in conjunction with rarity, suggest that fine-scale processes are likely to be important determinants of genetic diversity and structure in these species; however, no investigation of clonality has been published to date for this ecologically important genus.

Two species in particular, Lepidosperma sp. Mt Caudan and L. sp. Parker Range, are restricted to ironstone-rich soils of the Parker Range. These naturally rare species have persisted in this landscape for some time while maintaining diversity and tolerating considerable levels of inbreeding (Binks et al., 2015). Previous work confirms that both are outcrossing, although observations of poor seed set over recent years indicates that sexual reproduction is not regular for either species. Two consecutive seasons of good rainfall are required for flowering and seed development in Lepidosperma (Barrett, 2013) and this is likely to be the major limiting factor to sexual reproduction for both study species given that they co-occur in a low rainfall zone (Hopper, 1979). Their resilience in this landscape is thought to be attributed to a mixed strategy of sexual and asexual reproduction, which is consistent with geometric growth patterns, shared multilocus genotypes (MLGs) and high $F_{I S}$ values (Binks et al., 2015), although the extent of clonality in these species is unknown. Although both species occur in the same localized area and experience similar environmental conditions, their differing geographic distributions result in contrasting patterns of gene exchange and this may influence the relative balance of sexual and asexual reproduction for each species. Populations of $L$. sp. Mt Caudan are geographically clustered and well connected by gene flow (global $F_{\mathrm{ST}}=0.051$ ), while gene exchange is more restricted among the geographically disjunct populations of $L$. sp. Parker Range (global $F_{\mathrm{ST}}=0.277$ ) (Binks et al., 2015). Thus, while both species are rare and present indicators of asexual reproduction, the increased geographic isolation in $L$. sp. Parker Range may lead to a greater contribution of asexual reproduction as a result of limited effective gene exchange among isolated populations.
The aim of this study was to examine clonality in $L$. sp. Mt Caudan and $L$. sp. Parker Range in order to (i) confirm whether each species utilizes asexual reproduction and determine the extent and spatial architecture of clonality for each species and (ii) to compare the relative balance of sexual to asexual reproduction between species, to test the prediction that asexual reproduction should be more extensive in isolated than connected populations. Specifically, we estimate genotypic diversity and determine the spatial architecture of clonal patches by identifying shared MLGs within intensively sampled quadrats. In addition, we use spatial autocorrelation analyses at both the ramet and genet level to assess the impact of clonality on fine-scale genetic structure for each species.

\section{MATERIALS AND METHODS}

\section{Study species and sample collection}

Lepidosperma sp. Mt Caudan and L. sp. Parker Range are recognized phrase name species by the Western Australian Herbarium, each with a conservation status of Priority 1 (http://florabase.dpaw.wa.gov.au). Both species are restricted to the Parker Range region of southern Western Australia but have contrasting distributions in this localized area. The 11 known $L$. sp. Mt Caudan populations are geographically clustered in a small area (maximum $15 \mathrm{~km}$ apart). In contrast, $L$. sp. Parker Range is known from just six populations that are geographically isolated across $200 \mathrm{~km}$ (Figure 1). There have been no empirical studies of their ecology or mating systems to date, and we did not observe any viable seed set in the two years of this study to enable our own investigations. However, Lepidosperma are considered to be primarily outcrossing via windpollination and their andromonoecious arrangement of unisexual and bisexual flowers with dichogamous development presumably limits selfing (Barrett, 2013). Finally, the presence of elaiosomes suggests that seed dispersal may be facilitated by ants and birds.

In total, we collected approximately 600 samples across two populations of each species. Each pair of populations were roughly the same distance apart $(11 \mathrm{~km})$, but there were additional populations inbetween the two sampled L. sp. Mt Caudan populations (LMC2 and LMC3) that facilitate gene flow, whereas there were no populations between the two $L$. sp. Parker Range populations (LPR1 and LPR3) that experience restricted gene flow (Figure 1). Samples were collected within square quadrats, as recommended by ArnaudHaond et al. (2007), to maximize the genotypic diversity collected while minimizing possible edge effects. Within each population, two $15 \times 15 \mathrm{~m}^{2}$ quadrats were marked and a maximum of 75 samples were collected from each quadrat for genotyping; this resulted in the sampling of $90-100 \%$ of the distinct clumps within a given quadrat. The geographic location of each sample was recorded using a Differential Global Positioning System.

\section{DNA extraction and genotyping}

Leaf material was freeze-dried before genomic DNA was extracted using a $2 \%$ CTAB method (Doyle and Doyle, 1987), modified by adding 1\% polyvinylpyrrolodine to the extraction buffer. Nuclear microsatellite markers were developed specifically for each species as detailed in Binks et al. (2014). Of these, the most polymorphic loci (LMC 10, 18, 24, 25, 27, 38, 40 and LPR 03, $12,13,16,27,28,29,34)$ were chosen for genotyping. PCR products were visualized on a Biosystems 3730 Sequencer (Applied Biosystems, Foster City, CA, USA) and genotypes were scored manually using Genemapper v.3.7 (Applied Biosystems). Samples that did not amplify initially or were ambiguous in scoring were re-amplified and re-scored to eliminate errors in clone assignment and produce a complete data set.

\section{Clonal diversity and spatial structure}

We used GENCLONE v.2.0 (Arnaud-Haond and Belkhir, 2007) to assign samples within each quadrat to groups of identical MLGs. Before the full analysis, various permutation tests were performed to assess data quality. To determine whether replicates of the same MLG were the result of asexual reproduction, we estimated the probability of a given MLG arising from a distinct sexual reproductive event in a given population $\left(P_{\mathrm{GEN}}\right)$, and then the probability that $n$ incidences of an identical MLG were the result of $n$ distinct 


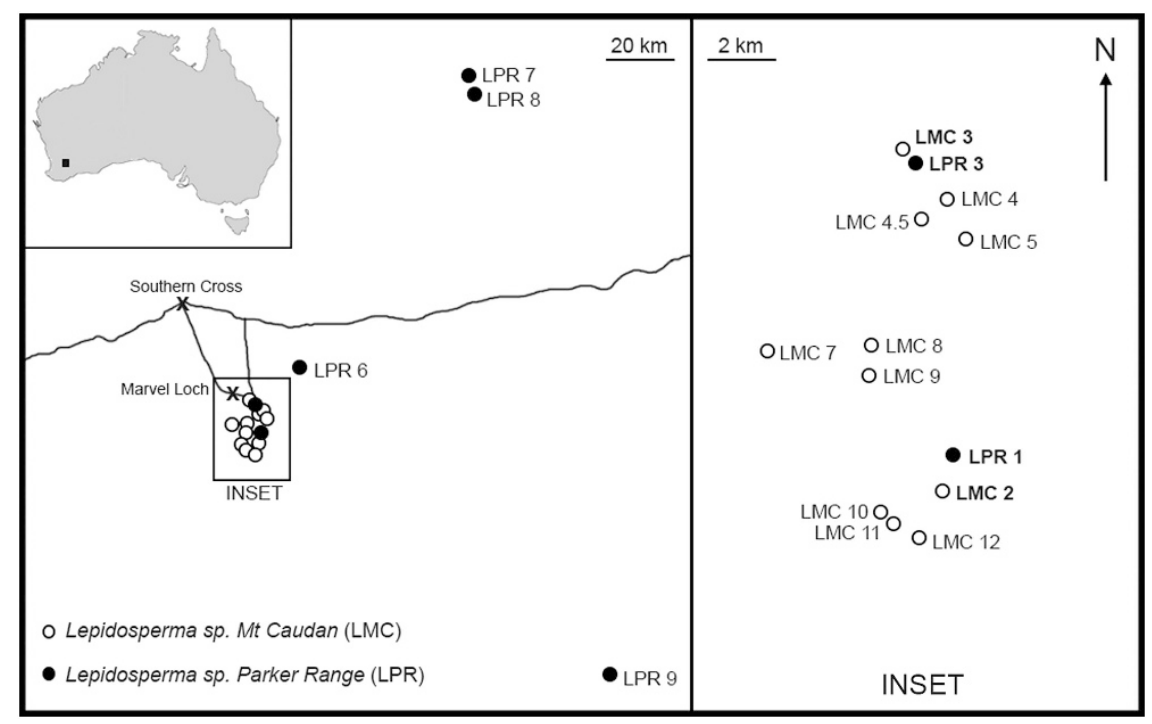

Figure 1 Location of all known populations of Lepidosperma sp. Mt Caudan (open circles) and Lepidosperma sp. Parker Range (closed circles) across the Parker Range, Western Australia. Population names in bold indicate those sampled in this study.

sexual reproductive events $\left(P_{\mathrm{SEX}}\right)$. Rejection of the null hypothesis $(P<0.001)$ provided confidence of asexual reproduction within distinct MLGs. We also used a re-sampling approach to assess the efficacy of our sampling densities and microsatellite loci to ensure we had sufficient power to resolve clonal diversity within a given quadrat. Finally, to account for possible somatic mutations or scoring errors, we grouped MLGs that differed at only one locus into multilocus lineages (MLLs) if $P_{\mathrm{SEX}}$ values, re-calculated after removing the locus in question, returned a significant likelihood of asexual origins. For subsequent analyses at the MLL level, mismatched loci were included but replicates were modified to a common genotype; MLLs with three or more ramets were reduced to their dominant genotype, while MLLs with just two ramets were assigned either the heterozygous genotype (if the other was homozygous) or the genotype with the most frequent allele at that locus, as per Chenault et al. (2011). For convenience, and because all MLLs were reduced to a common genotype for analysis, hereafter all references to MLGs includes any MLLs identified, unless specified. Finally, we indirectly assessed clonal diversity by measuring the genotypic richness $(R)$ of each quadrat as the proportion of plants with unique genotypes (Ellstrand and Roose, 1987): $R=(G-1) /(N-1)$ where $G$ is the number of distinct MLGs (genets) and $N$ is the number of sampled plants (ramets). All of the above analyses were performed using GENCLONE.

To assess the spatial arrangement of clonal patches, all MLGs were mapped and various parameters were estimated using GENCLONE: (i) clonal subrange, the maximum distance between any two ramets within the same MLG, as a measure of the size of genets and the spatial scale at which clonality no longer affects genetic structure, (ii) the aggregation index, testing whether genets are spatially aggregated or significantly intermingled, and (iii) the edge effect, testing whether diversity is unevenly distributed across each quadrat, to detect ineffective sampling that results in unique MLGs occurring more frequently on the edges of the sampling area. We also performed spatial autocorrelation analysis on the full (that is, ramet-level) data set of each species to assess the effect of clonality on fine-scale spatial genetic structure using SPAGEDI v.1.4 (Hardy and Vekemans, 2002). We applied Loiselle's kinship coefficient (Loiselle et al., 1995) across eight distance classes, each with upper limits of $0.4,0.8,1,2$, 4, 8, 12 and $15 \mathrm{~m}$. Significance was determined with 9999 permutations and jack-knifing across loci to calculate $95 \%$ confidence intervals and standard errors, respectively.

Fine-scale genetic diversity and structure

To determine genetic diversity and structure at fine-scales without the influence of clonality, we removed the replicates from each genet and continued analyses using a single copy of each unique genotype (that is, genet-level analysis). Using this data set, several genetic parameters were calculated for each quadrat in each population to allow comparisons with clonal parameters at the same scale. We used GENALEX v.6.5 (Peakall and Smouse, 2006) to calculate two measures of diversity, allelic richness and expected heterozygosity, as well as the inbreeding coefficient. We also used RMES (David et al., 2007) to estimate selfing rates from adult genotypes using the $g_{2}$ estimator of multilocus correlations in heterozygote deficiency. Finally, we repeated the spatial autocorrelation analyses for each species at the genet level but with fewer distance classes. Without replicate ramets, there were fewer pairwise combinations within the smaller classes, thus the genet-level analyses consisted of seven distance classes (that is, $0.5,1,2,4,8,12$ and $15 \mathrm{~m}$ ) to maintain adequate numbers of pairs within each class. For MLGs with two or more ramets, the central point of their individual locations was used for the analysis.

\section{RESULTS}

\section{Clonal diversity and spatial structure}

Permutation testing for the genotypic resolution of our study produced asymptotic trends in both accumulation curves; the gain from using additional loci or samples to detect the number of MLGs increased exponentially and stabilized well before the total number of loci and samples was reached. All replicate genotypes identified within MLGs were associated with $P_{\text {SEX }}$ values below the level of significance for sexual events (that is, $P<0.001$ ), thus we considered all replicates for both species to have arisen by asexual origins. The final data set included four incidences of MLLs for $L$. sp. Mt Caudan and three for L. sp. Parker Range, which were reduced to common genotypes for analysis. With confidence in asexual origins, MLGs with replicate samples can be referred to as genets with multiple ramets forming clonal patches, as distinct from MLGs with unique genotypes in single samples.

Asexual reproduction was uncommon in $L$. sp. Mt Caudan. Out of the 300 samples genotyped across all four quadrats, 267 MLGs were identified, of which $90 \%$ were unique genotypes from single samples (Figure 2a). Consequently, genotypic richness was high, with an average of $0.89 \pm 0.02$ across all quadrats (Table 1). Although infrequent, low levels of clonality were found in all quadrats (Figure 3) and patterns of clonal diversity were remarkably consistent across quadrats and populations (Table 1). Less than 10 genets occurred in a given quadrat and the few clonal patches present were always small, both in propagation size, with an average of $2.23 \pm 0.09$ 
ramets per genet, and in physical size, with an average clonal subrange of $1.3 \pm 0.11 \mathrm{~m}$ (Table 1). Figure 3 shows the spatial arrangement of clonal patches; ramets were significantly aggregated within genets and genets were distributed across quadrats with no particular pattern. Finally, there were no shared MLGs across quadrats or populations and no evidence for an edge effect within any quadrat.

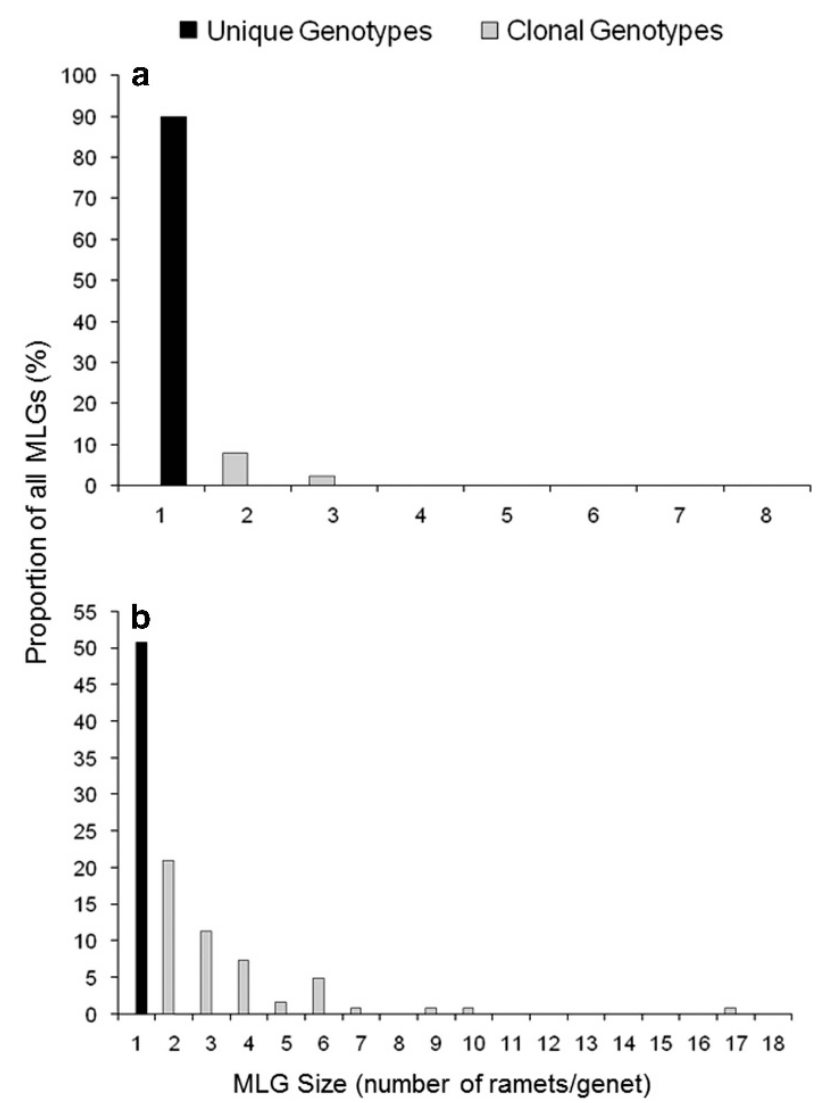

Figure 2 Overall frequency distribution of clone size among MLGs for each of (a) Lepidosperma sp. Mt Caudan and (b) Lepidosperma sp. Parker Range. Data are collated across all four quadrats for each species. Note that the axes on each figure have different scales.
In contrast to $L$. sp. Mt Caudan, clonality in L. sp. Parker Range was much more frequent and occurred over greater spatial scales. Overall, just $50 \%$ of the 124 MLGs identified had unique genotypes, with the remaining $50 \%$ exhibiting a leptokurtic trend in size distribution of few large clones and many smaller clonal patches (Figure 2b). Patterns of clonality were more variable among quadrats and populations than in $L$. sp. Mt Caudan and are most easily visualized in Figure 4. Greatest clonality was seen in LPR1 Q1, with the lowest genotypic richness $(R=0.14)$, the largest clonal patch in terms of propagation (17 ramets) and just one, single unique genotype among all samples genotyped. Interestingly, the second quadrat in the same population, LPR1 Q2 was the least clonal, with high genotypic richness $(R=0.77)$, small genet sizes (average of $2.42 \pm 0.24$ ramets per genet) and the highest number of single, unique genotypes (34 unique MLGs). Overall, patterns showed moderate levels of clonality for $L$. sp. Parker Range, with an average genotypic richness of $0.44 \pm 0.13$ (Table 1). No quadrat was dominated by a given genotype, each consisting of few large and many smaller genets. Ramets were found to be significantly aggregated within genets but more intermingling among genets can be seen than that found for $L$. sp. Mt Caudan (Figure 4). Genet size was variable in all quadrats with an average of $4.02 \pm 0.99$ ramets per genet and clonal subrange of $4.27 \pm 0.61 \mathrm{~m}$. Clonal patches tended to grow in circular or linear formations but the larger clonal patches often formed non-geometric patterns (Figure 4). Again, there was no evidence for an edge effect within any quadrat, and there were no shared MLGs across quadrats or populations.

Spatial autocorrelation at the ramet level showed significant spatial structuring at fine spatial scales for both species. For L. sp. Mt Caudan, the relationship between kinship and geographic distance was equally high at the shortest distances and rapidly became non-significant between 0.8 and $1 \mathrm{~m}$ (Figure 5a). In $L$. sp. Parker Range, kinship was high at the shortest distance class and progressively decreased with distance until the relationship became non-significant at $4 \mathrm{~m}$ (Figure 5b). These distances closely coincide with the average clonal subrange for each species.

Fine-scale genetic diversity and structure

A summary of genetic diversity at the genet level for all quadrats is given in Table 1 . Overall, allelic richness was greater in $L$. sp. Mt

Table 1 Summary of diversity parameters at the ramet and genet level of each quadrat sampled for Lepidosperma sp. Mt Caudan (LMC) and L. sp. Parker Range (LPR)

\begin{tabular}{|c|c|c|c|c|c|c|c|c|c|c|c|}
\hline \multirow{2}{*}{$\begin{array}{l}\text { Population/ } \\
\text { Quadrat }\end{array}$} & \multicolumn{6}{|c|}{ Ramet level } & \multicolumn{5}{|c|}{ Genet level } \\
\hline & $\mathrm{N}_{L}$ & $\mathrm{n}$ & $\mathrm{N}_{M L G}$ & $\mathrm{~N}_{G}$ & $\mathrm{~N}_{R / G}$ & $\mathrm{R}$ & $\mathrm{n}$ & $\mathrm{N}_{A}$ & $\mathrm{H}_{O}$ & $\mathrm{H}_{E}$ & $\mathrm{~F}_{/ S}$ \\
\hline LMC2 Q1 & 7 & 75 & 64 & 9 & $2.22(0.15)$ & 0.81 & 64 & $4.43(0.90)$ & $0.33(0.08)$ & $0.45(0.09)$ & $0.22(0.14)$ \\
\hline LMC2 Q2 & 7 & 75 & 69 & 6 & $2.00(0.00)$ & 0.92 & 69 & $4.84(1.17)$ & $0.89(0.07)$ & $0.50(0.05)$ & $0.27(0.11)$ \\
\hline LMC3 Q1 & 7 & 75 & 69 & 5 & $2.20(0.20)$ & 0.92 & 69 & $5.10(1.01)$ & $0.33(0.09)$ & $0.60(0.04)$ & $0.34(0.13)$ \\
\hline LMC3 Q2 & 7 & 75 & 65 & 7 & $2.43(0.20)$ & 0.87 & 65 & $5.28(1.14)$ & $0.45(0.08)$ & $0.59(0.05)$ & $0.21(0.11)$ \\
\hline Mean (s.e.) & & & $66.75(1.31)$ & & $2.23(0.09)$ & $0.89(0.02)$ & & $4.91(0.19)$ & $0.38(0.02)$ & $0.54(0.04)$ & $0.20(0.02)$ \\
\hline LPR1 Q1 & 8 & 56 & 9 & 8 & $6.88(1.63)$ & 0.15 & 9 & $2.70(0.56)$ & $0.11(0.10)$ & $0.42(0.10)$ & $0.54(0.23)$ \\
\hline LPR1 Q2 & 8 & 75 & 51 & 17 & $2.42(0.24)$ & 0.77 & 51 & $2.70(0.45)$ & $0.32(0.09)$ & $0.43(0.08)$ & $0.32(0.15)$ \\
\hline LPR3 Q1 & 8 & 75 & 39 & 17 & $3.12(0.45)$ & 0.51 & 39 & $3.83(0.56)$ & $0.41(0.10)$ & $0.55(0.06)$ & $0.24(0.14)$ \\
\hline LPR3 Q2 & 8 & 75 & 25 & 19 & $3.64(0.33)$ & 0.34 & 25 & $3.60(0.53)$ & $0.35(0.09)$ & $0.56(0.08)$ & $0.32(0.13)$ \\
\hline Mean (s.e.) & & & 31.00 (9.06) & & $4.02(0.99)$ & $0.44(0.13)$ & & $3.24(0.28)$ & $0.33(0.06)$ & $0.51(0.04)$ & $0.31(0.07)$ \\
\hline
\end{tabular}

Abbreviations: $F_{\mathrm{IS}}$, inbreeding coefficient; $H_{\mathrm{E}}$, expected heterozygosity; $H_{0}$, observed heterozygosity; $N_{\mathrm{A}}$, average number of alleles per locus; $N_{\mathrm{G}}$, number of clonal genets; $N_{\mathrm{L}}$, number of loci; $n$, number of samples; $N_{\mathrm{MLG}}$, number of multilocus genotypes (including lineages); $N_{\mathrm{R} / \mathrm{G}}$, average number of ramets per genet; $R$, genotypic richness. 


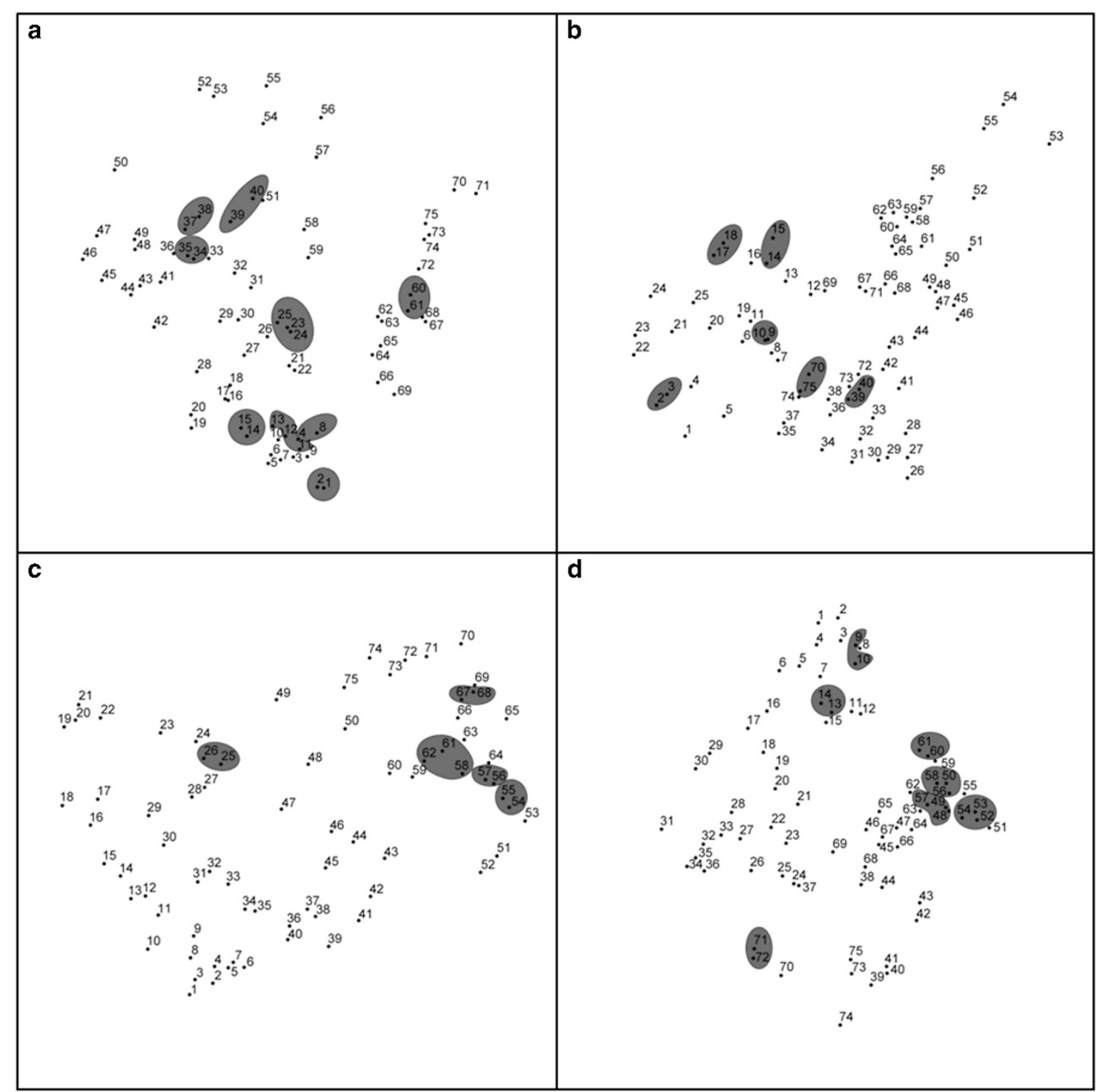

Figure 3 Size and distribution of clonal patches in populations of Lepidosperma sp. Mt Caudan. Each point represents a sample collected within $15 \times 15 \mathrm{~m}^{2}$ quadrats, (a) LMC2 Q1, (b) LMC2 Q2, (c) LMC3 Q1 and (d) LMC3 Q2. Ramets within the same genet are grouped by shading.

Caudan $\left(N_{\mathrm{A}}=4.91 \pm 0.19\right)$ than in $L$. sp. Parker Range $\left(N_{\mathrm{A}}=3.24 \pm\right.$ $0.28)$, although expected heterozygosity was similar $\left(H_{\mathrm{E}}=0.54 \pm 0.04\right.$ and $0.51 \pm 0.04$, respectively). Both species showed heterozygote deficiencies, but particularly L. sp. Parker Range, resulting in high inbreeding coefficients. Finally, selfing rates were not significantly different from zero for either species.

Spatial autocorrelation at the genet level showed contrasting patterns in each species. For $L$. sp. Mt Caudan, there was a significant effect at $1 \mathrm{~m}$ for ramets, but at the genet level, there was no longer a significant relationship between kinship and geographic distance at any distance class (Figure 5a). In L. sp. Parker Range, there was a significant effect up to $4 \mathrm{~m}$ for ramets, and at the genet level, there was a considerable decrease in the kinship coefficient at short distances; however, the relationship remained significant until the 2$\mathrm{m}$ distance class (Figure 5b), indicating some fine-scale isolation by distance in L. sp. Parker Range that is unrelated to asexual reproduction.

\section{DISCUSSION}

In rare plant populations or species, the balance between sexual and asexual reproduction is often skewed toward increased asexual reproduction as a result of environmental, genetic or geographic limitations to sexual reproduction (Honnay and Bossuyt, 2005; Silvertown, 2008). Here we confirm clonal propagation in two rare sedge species that both experience irregular seed set. Despite their common limitation to sexual reproduction, however, clonality was considerably more extensive in $L$. sp. Parker Range than its sympatric congener, L. sp. Mt Caudan, as demonstrated by the increased frequency, number of ramets per genet and spatial extent of clones. We suggest that this contrast in the relative balance of sexual and asexual reproduction is a result of further limitations to sexual reproduction in $L$. sp. Parker Range, via geographic isolation to effective pollen transfer among isolated populations, leading to the greater role of asexual reproduction (Wilcock and Neiland, 2002; Honnay and Bossuyt, 2005). This is consistent with other studies that 


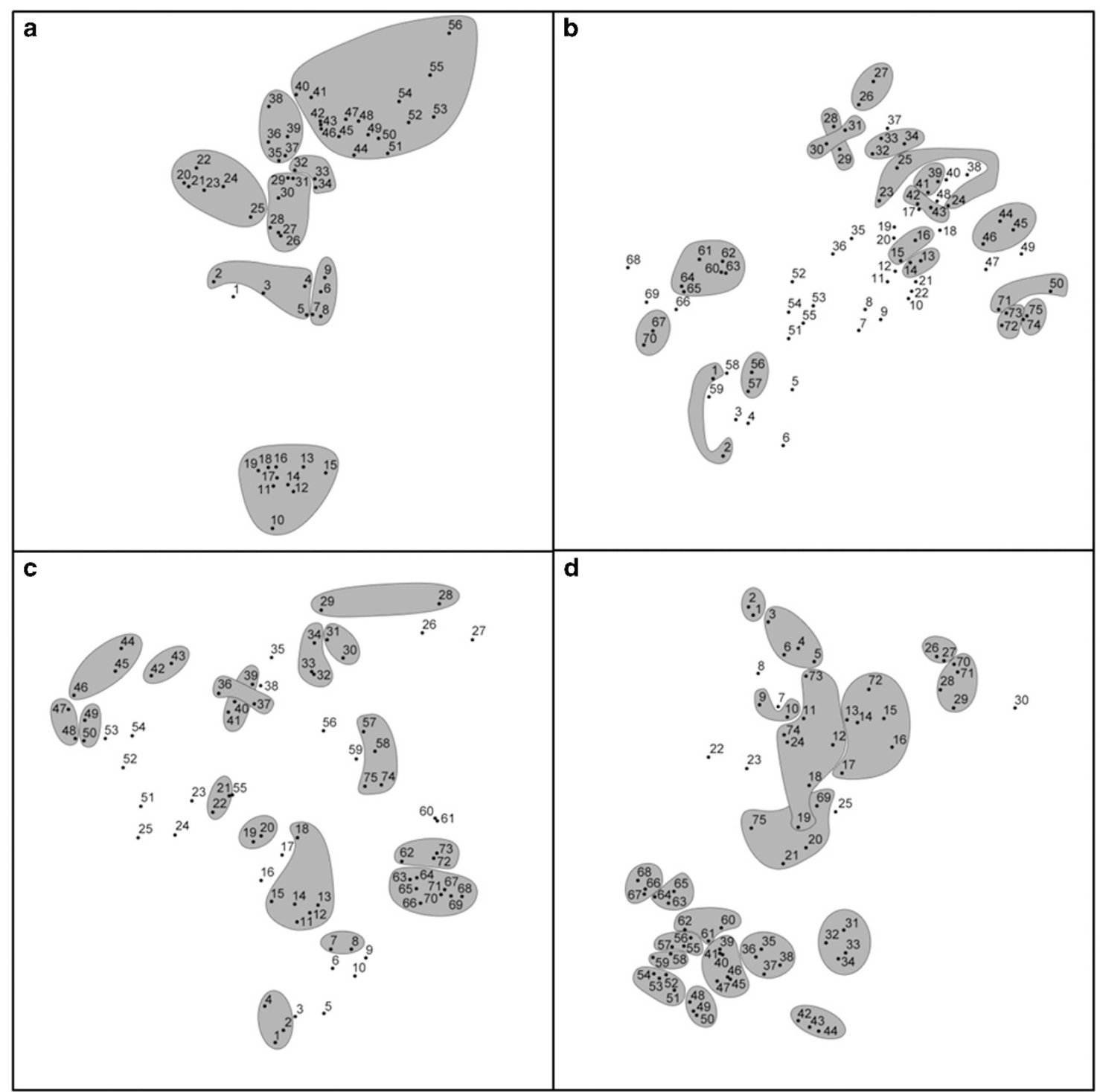

Figure 4 Size and distribution of clonal patches in populations of Lepidosperma sp. Parker Range. Each point represents a sample collected within $15 \times 15 \mathrm{~m}^{2}$ quadrats, (a) LPR1 Q1, (b) LPR1 Q2, (c) LPR3 Q1 and (d) LPR3 Q2. Ramets within the same genet are grouped by shading.

have found increased asexual to sexual reproduction in geographically isolated populations of plant species (Hooftman and Diemer, 2002; Rossetto et al., 2004) and is a likely a valuable means of persistence for such populations. Alternative environmental and genetic factors that commonly limit sexual reproduction in plants, such as a lack of pollinators or changes in ploidy (Eckert, 2002), are not relevant in these wind-pollinated, diploid species. These contrasting patterns of clonality in L. sp. Mt Caudan and L. sp. Parker Range have important implications for mating, fine-scale genetic structure and the conservation of these rare and localized species.

Overall, levels of genotypic diversity demonstrate that sexual reproduction is the primary means of reproduction for $L$. sp. Mt Caudan, while sexual and asexual reproduction are relatively equal contributors to reproduction in L. sp. Parker Range. The small, aggregated genets, when present in $L$. sp. Mt Caudan, have minimal influence on effective population size or genetic diversity and are unlikely to have an appreciable impact on mating opportunities in this species. Thus, while seed set is irregular, the geographic proximity of populations seems to ensure that, when good seasons of rainfall occur, sexual reproduction is sufficient to maintain the genetic integrity and persistence of $L$. sp. Mt Caudan in its narrow geographic range. In contrast, due to the larger and more numerous genets in the $L$. sp. Parker Range populations, effective population size is likely to be, on average, four times smaller than census counts, making $L$. sp. Parker Range even rarer than previously thought. However, there was no evidence of geitonogamous selfing, as is typically expected in clonal populations, particularly with large, aggregated genets (Vallejo-Marín et al., 2010). This is consistent with dichogamous flower development in the genus (Barrett, 2013) but may also reflect factors involved with self-incompatibility or postzygotic mortality that may be of interest for further study. Indeed, this apparent lack of selfing indicates that when sexual reproduction is successful, both species are predominantly outcrossing. These results also demonstrate the benefits of this mixed reproductive strategy for the isolated populations of $L$. sp. Parker Range; there appear to be minimal fitness costs in terms of inbreeding and the numerous, expanded genets allow for the on-going persistence of each population, while maximizing the chances of successful sexual reproduction in good seasons of rainfall. 
a

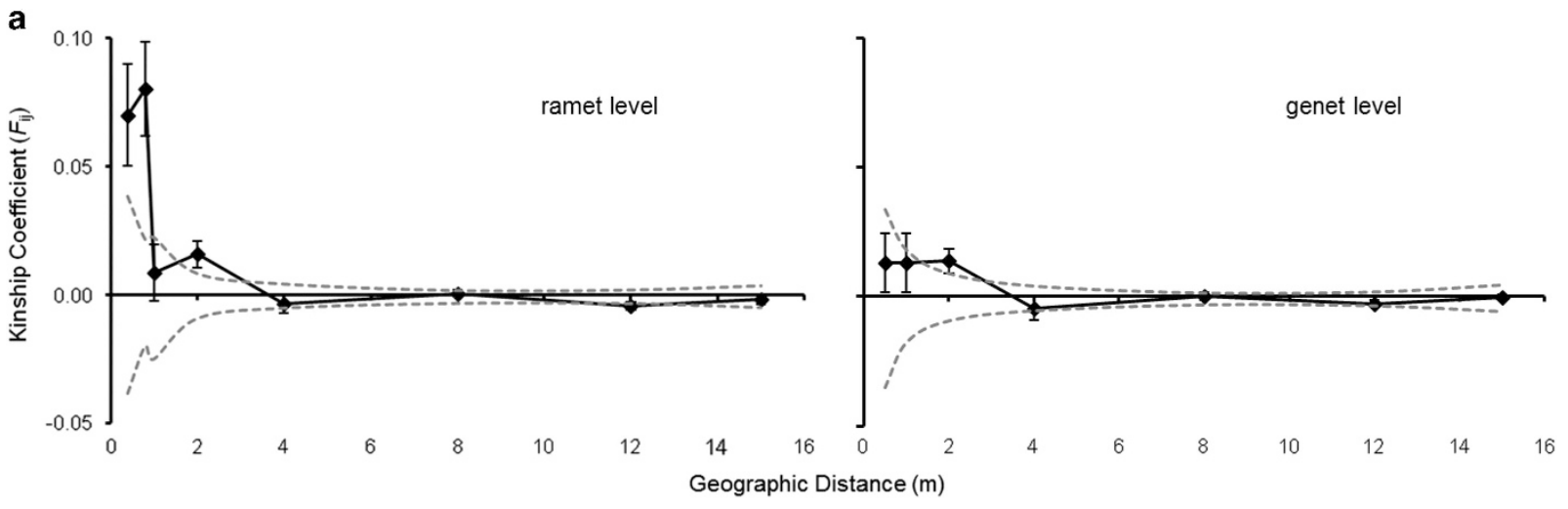

b

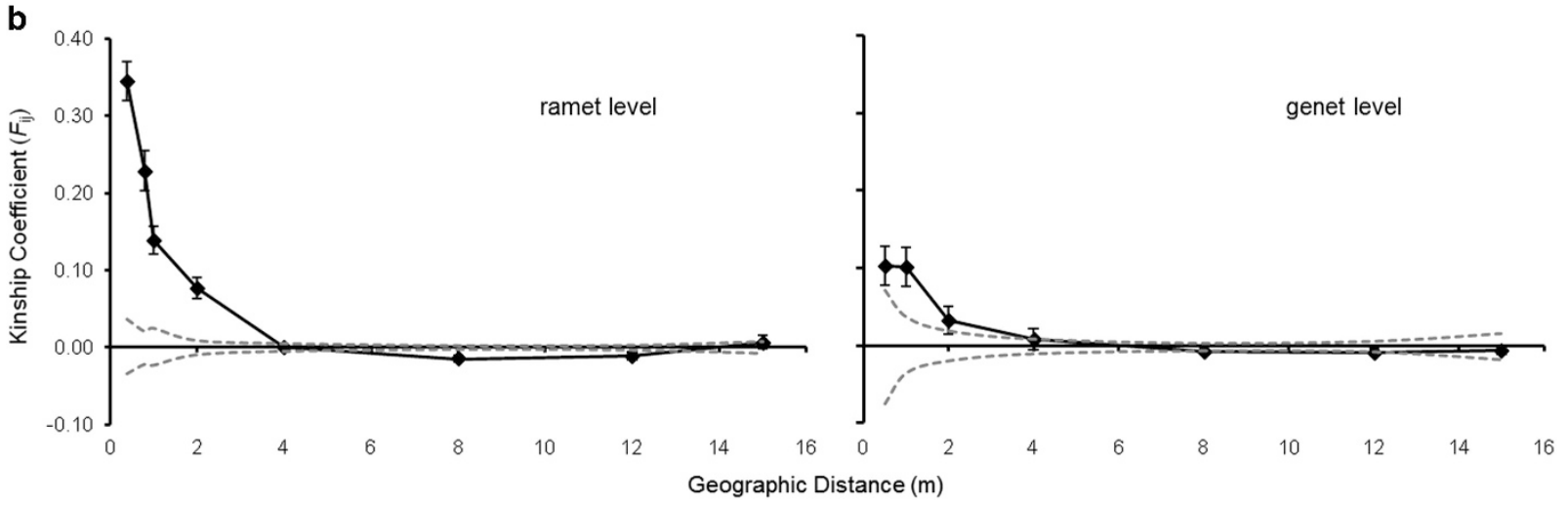

Figure 5 Spatial autocorrelation relationships between kinship and geographic distance for (a) Lepidosperma sp. Mt Caudan and (b) Lepidosperma sp. Parker Range, at both the ramet and genet levels. The permuted 95\% confidence interval (dashed lines) and jack-knifed s.e. bars are shown for each distance class.

Despite neither species being dominated by asexual reproduction, spatial autocorrelation analyses showed that clonal propagation is the major determinant of fine-scale genetic structure in both $L$. sp. Mt Caudan and L. sp. Parker Range. For L. sp. Mt Caudan, significant correlation was detected at the ramet level for distances $<1 \mathrm{~m}$; a relationship that became insignificant at the genet level, demonstrating that the majority of spatial structure within these populations is attributed to the proximity between clonemates. This highlights that even low levels of clonality can significantly impact fine-scale genetic structure in plant species. The lack of structure at the genet level likely reflects the high levels of connectivity among the $L$. sp. Mt Caudan populations (Binks et al., 2015) with indiscriminate wind pollination preventing the development of fine-scale patterns. For $L$. sp. Parker Range, our results show that both asexual and sexual reproduction contribute to fine-scale genetic structure. Although clonality affected spatial structure up to $4 \mathrm{~m}$, its removal revealed significant structure up to $2 \mathrm{~m}$ at the genet level. Such fine-scale isolation by distance may arise by selfing or restricted seed dispersal. Given that we found no evidence for selfing and Lepidosperma seeds exhibit elaiosomes (Barrett, 2013), this fine-scale genetic structure in $L$. sp. Parker Range is most likely due to restricted seed dispersal by ants.

The impact of clonality on fine-scale structure was strengthened by the significant aggregation of ramets within clonal patches for each species. Such aggregation is indicative of phalanx-type clonal growth, which is consistent with our observations of geometric growth patterns in both $L$. sp. Mt Caudan and L. sp. Parker Range, and with the short rhizomes exhibited by the closest phylogenetic relatives of each species, L. gibsonii and L. ferriculmen, respectively (Barrett, 2007).
However, while not significant, there was more intermingling of distinct genets in the more clonal $L$. sp. Parker Range, and geometric growth patterns were often lost in larger genets. These spatial patterns of non-random ramet growth may be a result of microhabitat heterogeneity (for example, Gao et al., 2012; Listl and Reisch, 2012) or competition among neighbouring genets or other species (for example, Benot et al., 2013; Xue et al., 2013) affecting the directions of ramet growth.

It is important to recognize that this study assessed just two populations for each species; although given their rarity, this is not a poor representation of either species. Although the frequency and size of clones varied among quadrats and populations, particularly for L. sp. Parker Range, the overall pattern of clonality was fairly consistent for each species so we are confident in extrapolating that the general patterns of clonality, being moderate in $L$. sp. Parker Range and low in L. sp. Mt Caudan, persist throughout their ranges. More specifically, these paired populations were chosen for their comparable distances apart and similar environmental conditions between species. For L. sp. Mt Caudan, these represent marginal populations from their range where clonality is typically more common (Silvertown, 2008) so clonality may be even less common in the central populations of $L$. sp. Mt Caudan. In turn, although all L. sp. Parker Range populations are considerably isolated, the populations sampled in this study are central to the species' range. We would therefore predict that the extent of clonality in the marginal, more isolated populations of $L$. sp. Parker Range (for example, LPR9; Figure 1) may present the more extreme levels of clonality for the species, which may be of interest for further study. 


\section{CONCLUSIONS}

In summary, we have demonstrated that both Lepidosperma species have mixed reproductive capacities but asexual reproduction contributes far more significantly to the mating system of $L$. sp. Parker Range than $L$. sp. Mt Caudan. We conclude that this contrast in the relative balance of sexual and asexual reproduction is largely a consequence of their contrasting geographic distributions and relative capacities for pollen dispersal. This highlights that the prevalence of asexual reproduction in rare species is contextual and is more likely to occur with the combined aspects of rarity and isolation, rather than generalized rarity in itself. In addition, these results provide vital knowledge for the management of two ecologically important sedges. Both species appear to be stable at present, their differing reproductive strategies allowing each to persist and maintain genetic diversity in their narrow geographic ranges. However, any environmental change may require conservation intervention, particularly for the more isolated and clonal $L$. sp. Parker Range. In order to maximize genotypic diversity, any collections of material for intervention should collect samples at least $1 \mathrm{~m}$ apart for $L$. sp. Mt Caudan and $4 \mathrm{~m}$ apart for L. sp. Parker Range. Finally, future research should investigate the mating systems of these species, particularly in terms of outcrossing and self-compatibility, should an opportunity of good conditions for sexual reproduction present itself.

\section{DATA ARCHIVING}

Microsatellite sequence details are available on GenBank and all raw genotypic data are available in the Dryad repository: http://dx.doi.org/ 10.5061/dryad.j94s2.

\section{CONFLICT OF INTEREST}

The authors declare no conflict of interest.

\section{ACKNOWLEDGEMENTS}

This research was supported by Cazaly Resources Limited. We thank Jim Williams for assistance with population surveys and sample collection.

Albert T, Raspé O, Jacquemart A-L (2008). Influence of clonal growth on selfing rate in Vaccinium myrtillus L. Plant Biol 10: 643-649.

Arnaud-Haond S, Belkhir K (2007). GENCLONE: a computer program to analyse genotypic data, test for clonality and describe spatial clonal organization. Mol Ecol Notes 7 15-17.

Arnaud-Haond S, Duarte CM, Alberto F, Serrão EA (2007). Standardizing methods to address clonality in population studies. Mol Ecol 16: 5115-5139.

Balloux F, Lehmann L, de Meeûs T (2003). The population genetics of clonal and partially clonal diploids. Genetics 164: 1635-1644.

Barrett RL (2007). New species of Lepidosperma (Cyperaceae) associated with banded ironstone in southern Western Australia. Nuytsia 17: 37-60.

Barrett RL (2013). Ecological importance of sedges: a survey of the Australasian Cyperaceae genus Lepidosperma. Ann Bot 111: 499-529.
Benot M-L, Bittebiere A-K, Ernoult A, Clément B, Mony C (2013). Fine-scale spatial patterns in grassland communities depend on species clonal dispersal ability and interactions with neighbours. J Ecol 101: 626-636.

Binks RM, Gardner MG, Millar MA, Byrne M (2014). Characterization and cross-amplification of novel microsatellite markers for two Australian sedges, Lepidosperma sp. Mt Caudan and L. sp. Parker Range (Cyperaceae). Conserv Genet Resour 6: 333-336.

Binks RM, Millar MA, Byrne M (2015). Not all rare species are the same: contrasting patterns of genetic diversity and population structure in two narrow-range endemic sedges. Biol J Linn Soc 114: 873-886.

Byrne M, Coates DJ, Forest F, Hopper SD, Krauss SL, Sniderman K et al. (2014). A diverse flora - species and genetic relationships. In: Lambers $\mathrm{H}$ (ed). Plant Life on the Sandplains in Southwest Australia: A Global Biodiversity Hotspot. UWA Press: Perth, Western Australia, Australia. pp 81-99.

Charpentier A (2002). Consequences of clonal growth for plant mating. Evolution 15: 521-530.

Chenault N, Arnaud-Haond S, Juteau M, Valade R, Almeida J-L, Villar M et al. (2011). SSR-based analysis of clonality, spatial genetic structure and introgression from the Lombardy poplar into a natural population of Populus nigra L. along the Loire River. Tree Genet Genomes 7: 1249-1262.

David P, Pujol B, Viard F, Castella V, Goudet J (2007). Reliable selfing rate estimates from imperfect population genetic data. Mol Ecol 16: 2474-2487.

Doyle JJ, Doyle JL (1987). A rapid DNA isolation procedure for small quantities of fresh leaf tissue. Phytochem Bull 19: 11-15.

Eckert CG (2002). The loss of sex in clonal plants. Evol Ecol 15: 501-520.

Ellstrand NC, Elam DR (1993). Consequences of small population size: implications for plant conservation. Annu Rev Ecol Syst 24: 217-242.

Ellstrand NC, Roose ML (1987). Patterns of genotypic diversity in clonal plant species. Am J Bot 74: 123-131.

Gao Y, Xing F, Jin Y, Nie D, Wang Y (2012). Foraging responses of clonal plants to multipatch environmental heterogeneity: spatial preference and temporal reversibility. Plant Soil 359: 137-147.

Hardy OJ, Vekemans X (2002). SPAGEDI: a versatile computer program to analyse spatial genetic structure at the individual or population levels. Mol Ecol Notes 2: 618-620.

Honnay O, Bossuyt B (2005). Prolonged clonal growth: escape route or route to extinction? Oikos 108: 427-432.

Hooftman DAP, Diemer M (2002). Effects of small habitat size and isolation on the population structure of common wetland species. Plant Biol 4: 720-728.

Hopper SD (1979). Biogeographical aspects of speciation in the southwest Australian flora. Annu Rev Ecol Syst 10: 399-422.

Listl D, Reisch C (2012). Spatial genetic structure of the sedge Carex nigra reflects hydrological conditions in an alpine fen. Arctic, Antarct Alp Res 44: 350-358.

Loiselle BA, Sork VL, Nason J, Graham C (1995). Spatial genetic structure of a tropical understory shrub, Psychotria officinalis (Rubiaceae). Am J Bot 82: 1420-1425.

Lovett Doust L (1981). Population dynamics and local specialization in a clonal perennial (Ranunculus repens). I The dynamics of ramets in contrasting habitats. J Ecol 69 743-755.

Meloni M, Reid A, Caujapé-Castells J, Marrero A, Fernández-Palacios JM, Mesa-Coelo RA et al. (2013). Effects of clonality on the genetic variability of rare, insular species: the case of Ruta microcarpa from the Canary Islands. Ecol Evol 3: 1569-1579.

Myers N, Mittermeier RA, Mittermeier CG, da Fonseca GA, Kent J (2000). Biodiversity hotspots for conservation priorities. Nature 403: 853-858.

Ohsako T (2010). Clonal and spatial genetic structure within populations of a coastal plant, Carex kobomugi (Cyperaceae). Am J Bot 97: 458-470.

Peakall R, Smouse PE (2006). GENALEX 6: genetic analysis in Excel. Population genetic software for teaching and research. Mol Ecol Notes 6: 288-295.

Rossetto M, Gross CL, Jones R, Hunter J (2004). The impact of clonality on an endangered tree (Elaeocarpus williamsianus) in a fragmented rainforest. Biol Conserv 117: 33-39.

Silvertown J (2008). The evolutionary maintenance of sexual reproduction: evidence from the ecological distribution of asexual reproduction in clonal plants. Int J Plant Sci 169: $157-168$.

Vallejo-Marín M, Dorken ME, Barrett SCH (2010). The ecological and evolutionary consequences of clonality for plant mating. Annu Rev Ecol Evol Syst 41: 193-213.

Wilcock C, Neiland R (2002). Pollination failure in plants: why it happens and when it matters. Trends Plant Sci 7: 270-277.

Xue W, Huang L, Dong B-C, Zhang M-X, Yu F-H (2013). Patchy distributions of competitors affect the growth of a clonal plant when the competitor density is high. PLoS One 8 : e78221. 